\title{
OPTICAL VARIABILITY OF TWENTY-TWO QUASI-STELLAR OBJECTS
}

\author{
R. J. ANGIONE* and H. J. SMITH \\ University of Texas, Austin, Texas, U.S.A.
}

\begin{abstract}
Light fluctuations have been found in all 22 QSOs studied by measurement of plates from the Harvard collection, which cover the last eighty years. The conclusions of this study are: (i) There appear to be at least three general classes of variation: (a) erratic, small-amplitude variations, (b) erratic, large-amplitude variations, and (c) slow quasi-periodic variations, e.g. as in 3C 273; (ii) No significant differences were detected between the rates of rise and decline of luminosity; (iii) Definite secular trends over at least 50 years were found in 5 QSOs; (iv) No simple clearcut periods greater than one year have been found; (v) There may be a trend of decreasing amplitude of fluctuations in apparent magnitude with increasing luminosity.
\end{abstract}

Twenty-five objects, of which 22 proved to be QSOs, were selected for historical photometric investigation, solely on the basis of their relatively bright apparent magnitudes (brighter than $m=16.6$ ). The Harvard historical plate collection covering the last eighty years was searched for plates containing images of any of these objects; where possible, B magnitudes of the QSOs were measured with an iris photometer against photoelectrically-determined comparison star sequences in the immediate vicinity of each (Angione, 1970), or estimated visually against the sequence if iris photometry was not feasible on the particular plate. Various criteria establish the standard deviation of the photographic B magnitudes so determined as about $0.10 \mathrm{mag}$. An average of 74 plates per object was available.

These historical data can be used to ask at least six important questions concerning the behavior and nature of QSOs:

(1) What proportion of QSOs vary 'significantly' (by more than 0.1 mag.) over time scales ranging up to decades?

(2) Do the types of variations show any consistent differences or groupings?

(3) Do QSOs really 'flare'?

(4) Do QSOs show secular trends - individually or collectively?

(5) Is periodic behavior indicated?

(6) Do QSOs collectively demonstrate an amplitude-luminosity relation?

The remainder of this note presents brief summaries of our new information bearing on each of these questions. For detailed data and discussions of these results, see Angione (1970).

\section{Percentage of QSOs Found to Vary}

All 22 of the true QSOs in this sample were found to vary significantly over the 50-70 yr of available data. Standard deviations of individual QSO magnitudes about their means ranged from 0.13 to 0.62 mag. ( $3 \mathrm{C} 48$ and $3 \mathrm{C} 273$, both well-known variables,

* Now at San Diego State College. 
had respectively $\sigma=0.15$ and 0.21 ). After the completion of the data-taking we found that the original list of objects studied in the Harvard plate stacks contained two 'QSOs' (Ton 883 and $3 \mathrm{C} 68.2 \mathrm{~W}$ ) which were reported to be mis-identified stars, presumably constant in brightness. It is gratifying that these objects did prove to be

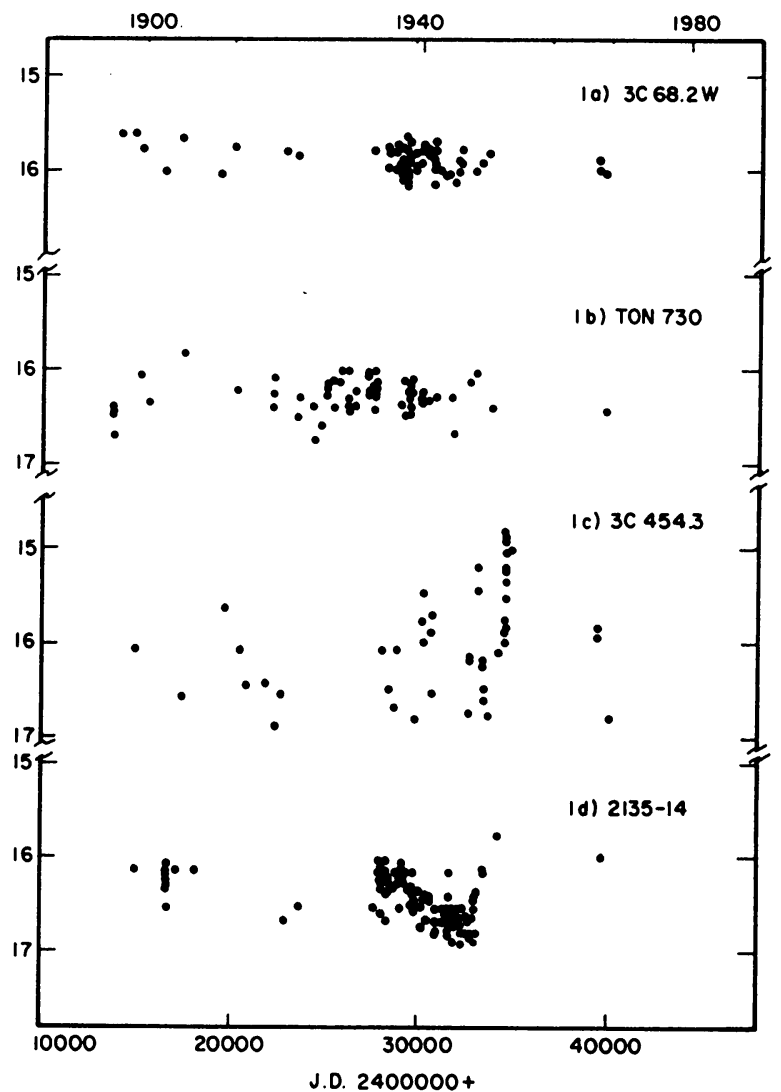

Fig. 1. Typical photometric data: 1a: A non-variable star, indicating the size and nature of the random observational errors in the material. 1b: A low-amplitude QSO. 1c: A large-amplitude 'violent' QSO. 1d: A 'coherently' variable QSO.

constant in the photometry of this program, with individual-measurement standard deviations of about 0.10 mag.; the 'light curve' of $3 \mathrm{C} 68.2 \mathrm{~W}$ is shown in Figure 1a.

\section{Types of Variations}

There appear to be at least three general classes of variation, at least to the resolution available in these data: (a) relatively rapid, erratic, small-amplitude variations (Figure 1b); (b) rapid, erratic, large-amplitude variations (Figure 1c); (c) relatively slow, correlated, quasi-periodic variations like those of 3C 273 (Figure 1d), with more erratic behavior apparently superimposed. In our data there are no well-defined 
connecting links between classes $a$ and $b$; the distribution may be truly bi-modal (see also Figure 4).

\section{Reality of 'Flares'?}

To test whether the rates of increase of brightness are significantly different from those of decline, the frequencies of observed changes by a given magnitude in a given interval of time were collected from the entire body of data. Figure 2 shows the

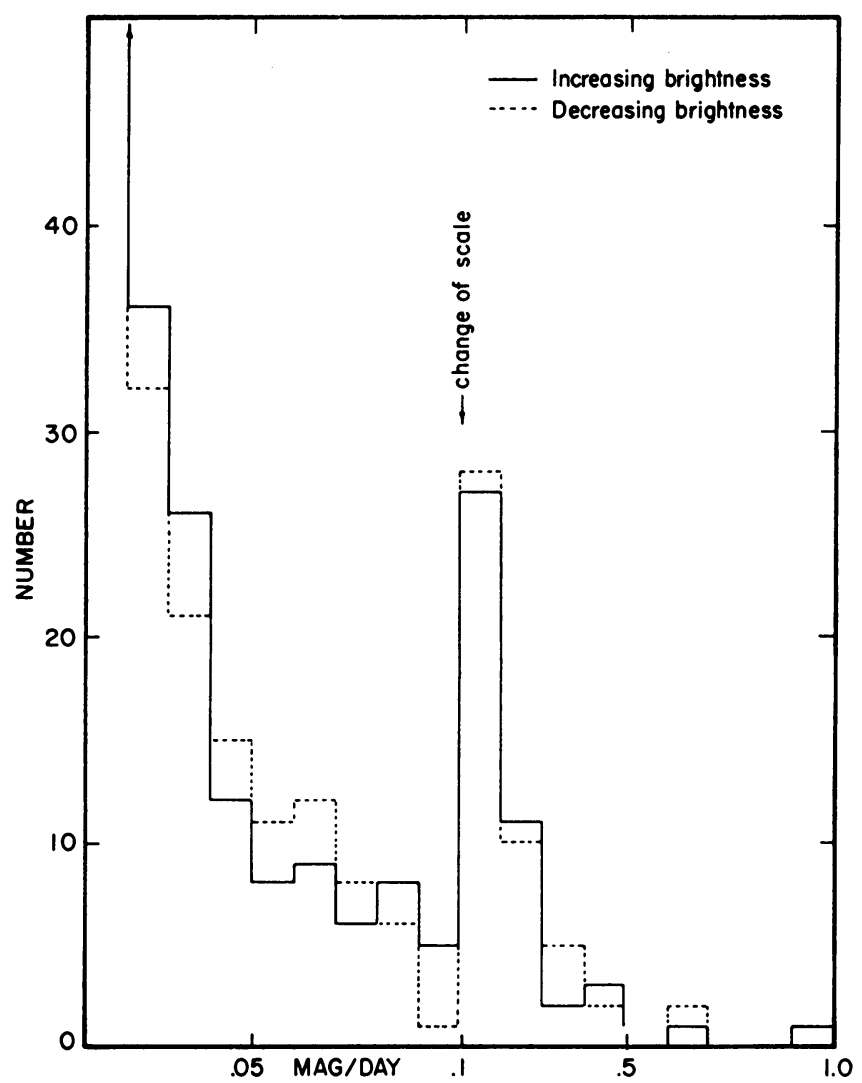

Fig. 2. Comparison of rates of increase and rates of decrease of QSO brightness.

interesting result that within the limits of the present measurements the QSOs display the same average frequency of rates of decline as rates of rise over all the ranges accessible to our tests. While many quasar models can undoubtedly be adjusted to fit such behavior, it is an expected aspect of at least two: a large rotating mass (galactic pulsar), or a gravitational lens effect.

\section{Secular Trends}

Five of the QSOs individually showed secular trends persisting for more than fifty years for which a linear least-squares fit indicated a $4 \sigma$ or greater confidence level: 
2135-14 and 3C 232 declining at rates respectively of 0.58 and 0.80 mag. per century; Ton 256, 0405-12, and 0237-23 brightening by $1.58,1.04$, and 0.64 mag. per century respectively. These numbers would be consistent with major changes in the behavior of about $25 \%$ of QSO cores over time scales of $10^{3} \mathrm{yr}$; alternatively the time-scale of fluctuation about some average brightness may simply be longer than we have yet been able to observe.

One may also question whether the quasar phenomenon in general leads to an average detectable systematic increase or decrease in brightness over time-scales of the order of a century; such a systematic trend might be detected in the mean behavior of a large number of objects. The average slope for the entire group of QSOs corresponds to a brightening at about 0.12 mag. per century (slightly dependent on weighting factors used). Since a few high or low observations, especially in the early years when good plates were rare, can substantially affect this result, and since even a single case of a relatively steep slope can strongly bias the average, we do not believe that these results establish any general trend in the optical brightness of QSOs.

\section{Periodic Behavior}

An improved form of periodogram analysis by Dr. Terence J. Deeming of the University of Texas Astronomy Department was used to analyze the original data without

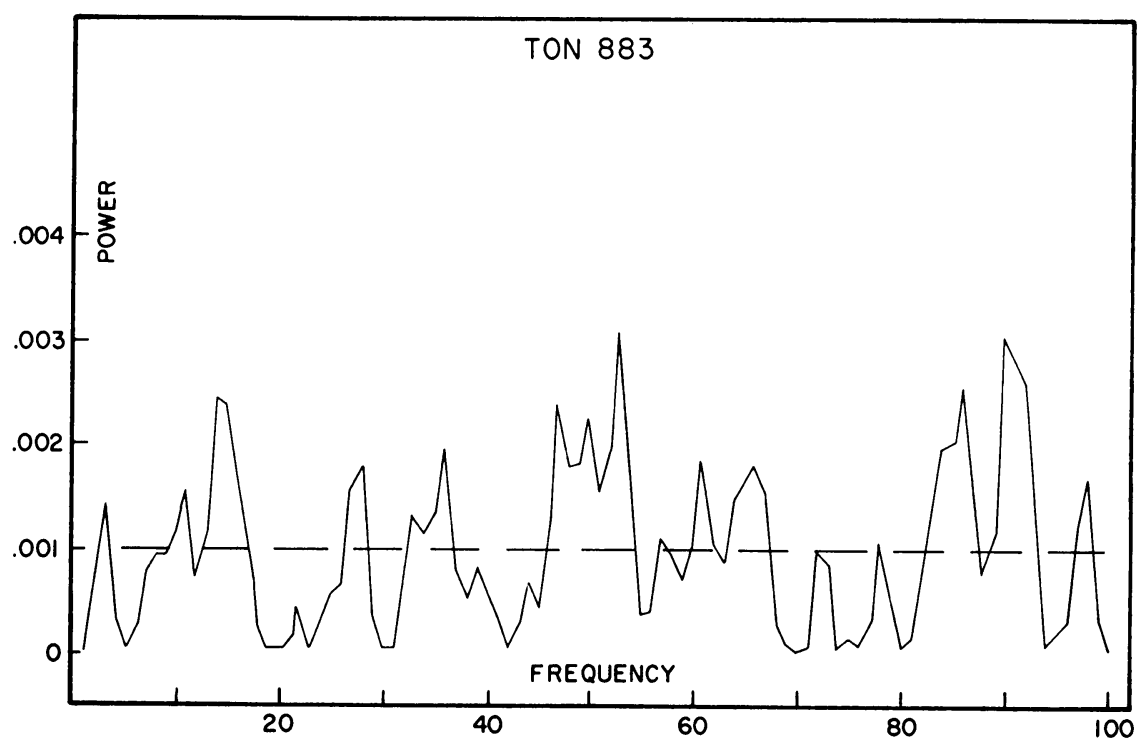

Fig. 3a.

smoothing to uniform intervals of time. Periodograms were formed for each of the light curves; as expected, random fluctuations are shown by the data for the stars inadvertently included in the program (e.g. Figure 3a). In three of the QSOs (3C 273, 2135-14, 0405-12; Figures 3b, 3c, 3d) about half of the spectral energy is concentrated 


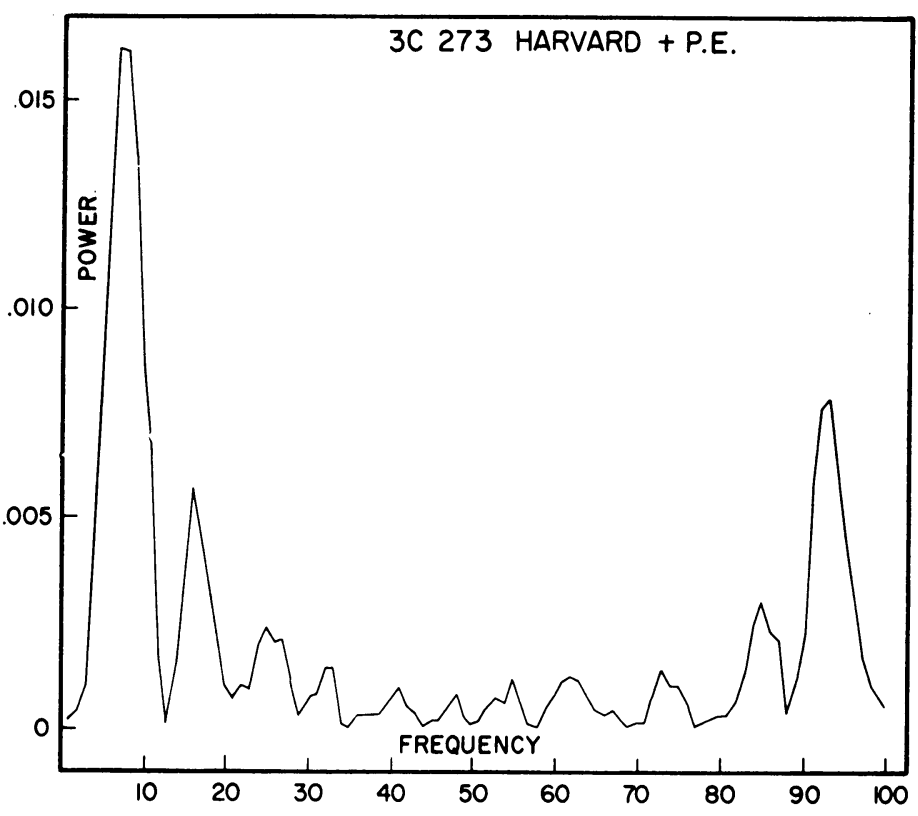

Fig. 3b.

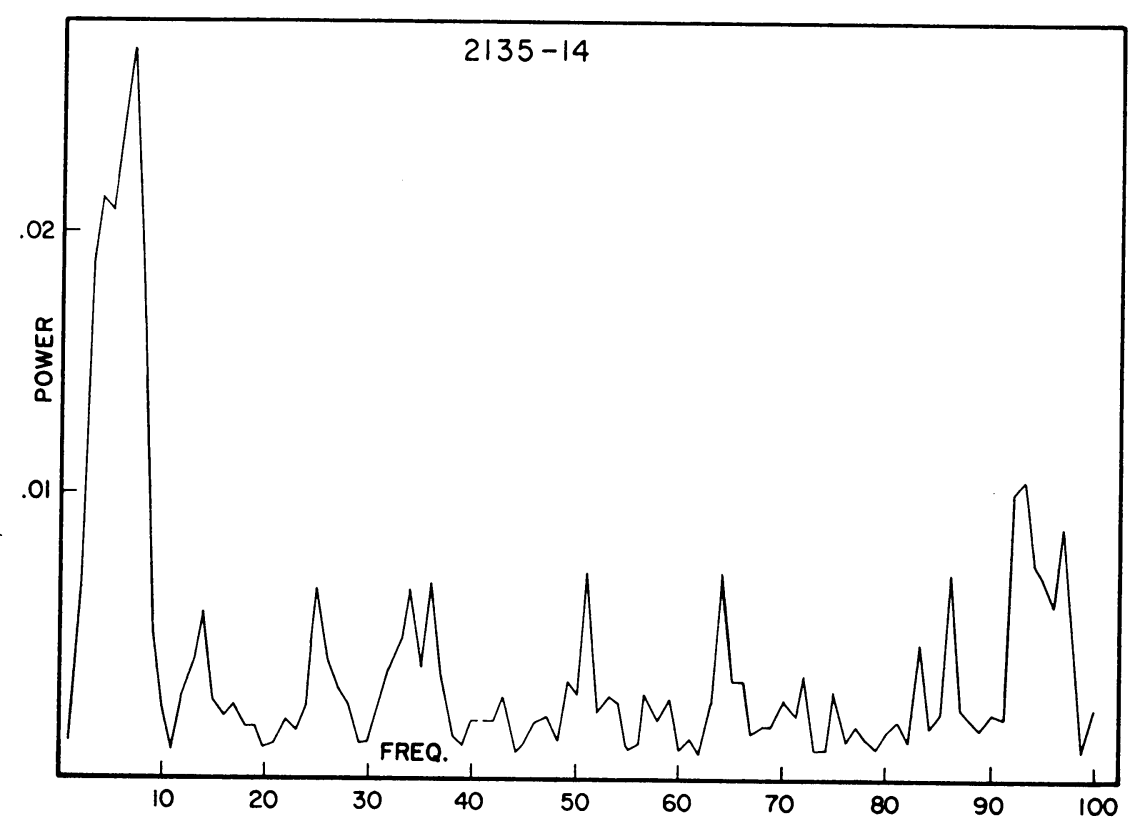

Fig. 3c. 


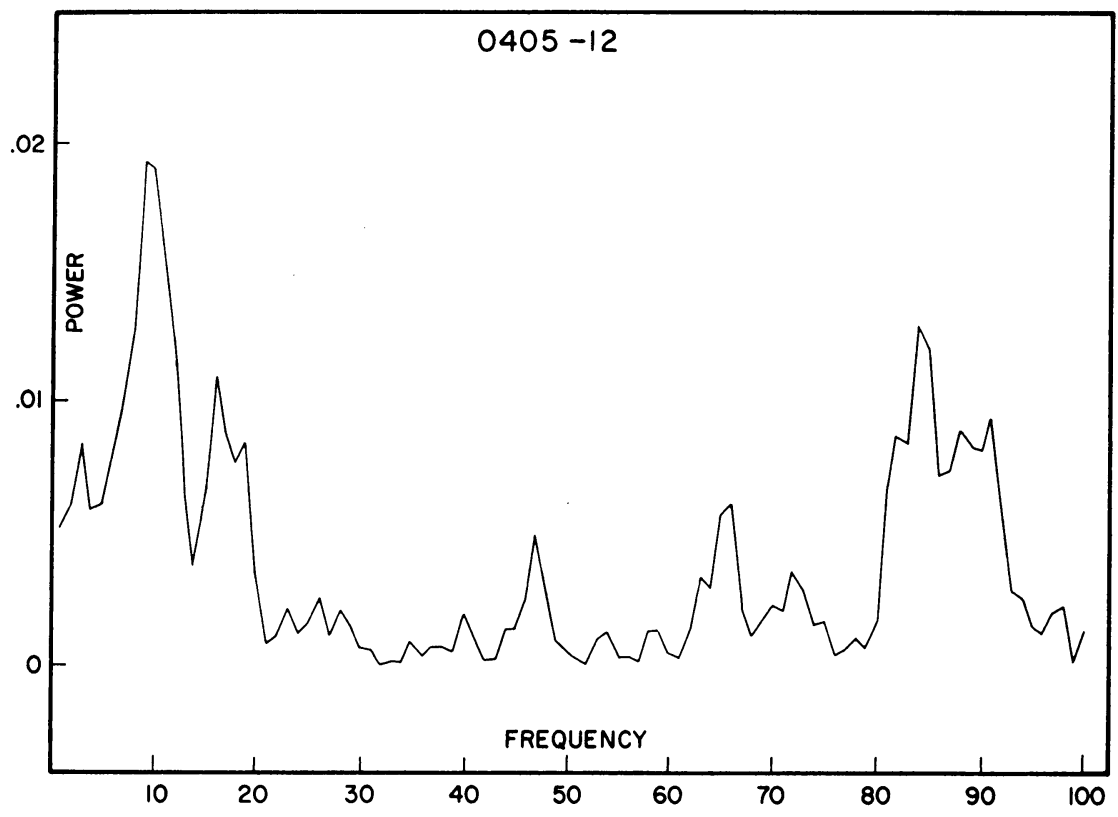

Fig. 3d.

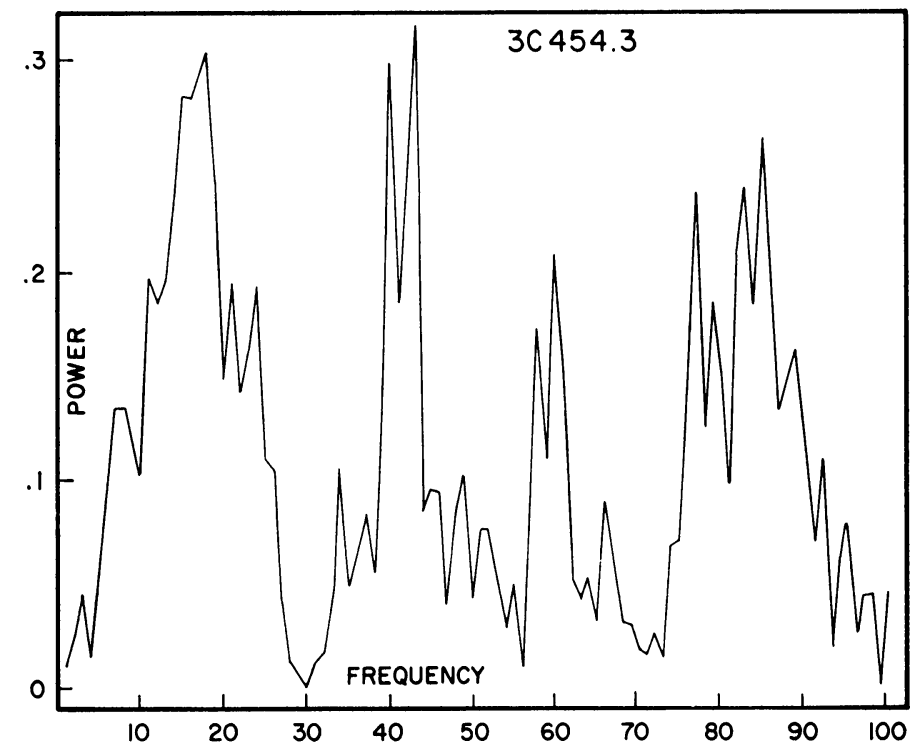

Fig. 3a-e. Typical power spectra. The abscissa frequency unit is $0.01 \mathrm{yr}^{-1}$ (cycles per century). Each diagram thus covers periods from $100 \mathrm{yr}$ to $1 \mathrm{yr}$. Long-term trends were first removed by fitting a straight line through the data. (a) A non-variable star Ton 883. (b), (c), (d) QSOs of the 3C 273 type.

(e) A violent QSO, 3C 454.3. 
in the interval around 11 to $13 \mathrm{yr}$. The figures also show that in each of these QSOs there is appreciable power at about $1.2 \mathrm{yr}$. $3 \mathrm{C} 273$, for which the data are much the most extensive, appears to be the most nearly periodic object in the entire group of QSOs. The majority of the periodograms are noisy (e.g. 3C 454.3, Figure 3e), although with some suggestion of harmonics. In summary, no simple clearcut periods greater than one year have been found, but nearly all the QSOs show several regions of frequency in which there are substantial concentrations of variational power.

\section{Amplitude-Luminosity Relation}

Variability offers a test, informally suggested by J. Wheeler, whether individual quasars are fundamentally multiple in nature (e.g. collections of large numbers of randomly flaring objects such as supernovae), or are essentially single coherent objects. The criterion follows from a simple correlation of amplitude of variation vs absolute magnitude. On a cosmological interpretation of the redshifts, the absolute magnitudes

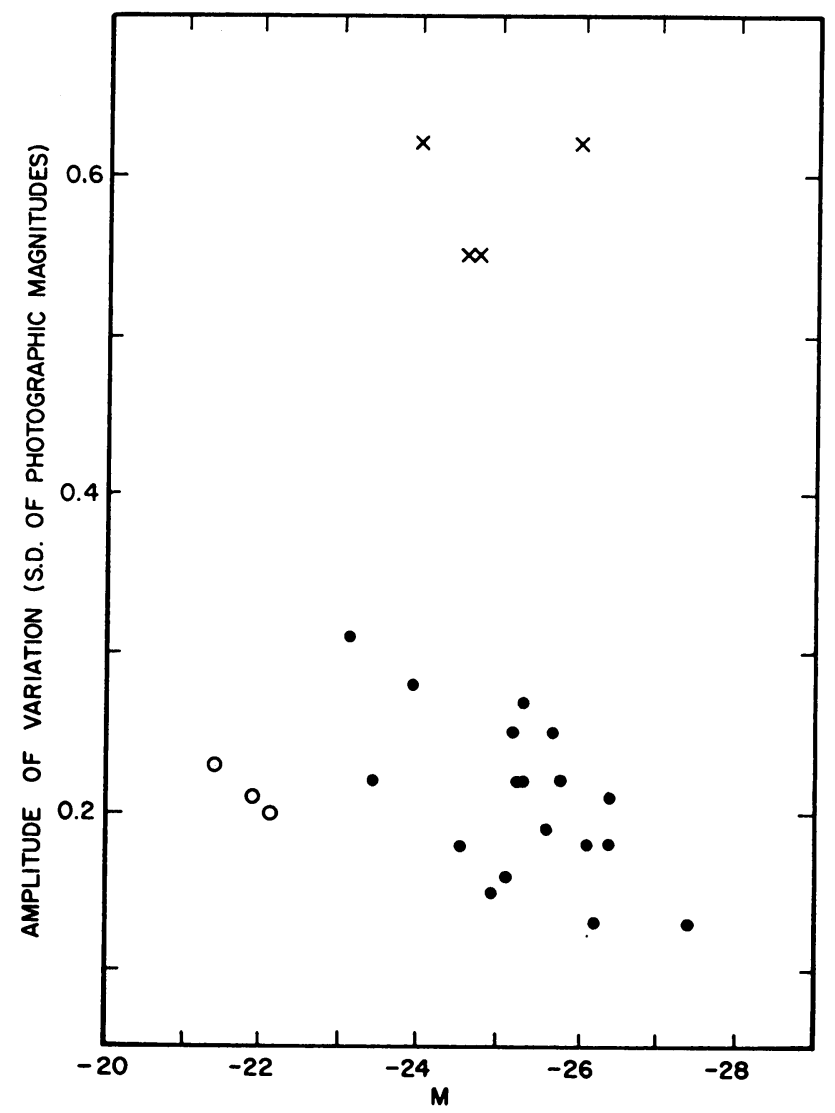

Fig. 4. Amplitude-luminosity relation for the objects in this study. Crosses denote the 'violent' QSOs, 3C 279, 345, 446 and 454.3. Open circles denote low redshift, low-luminosity objects probably of Seyfert or N-galaxy nature. Filled circles denote the remaining more ordinary QSOs. 
of the QSOs follow from their apparent magnitudes and from an assumed Hubble constant. If the total luminosity of each QSO represents the sum of a number of separate randomly-occurring events of a relatively similar character throughout the universe (e.g. the explosion of very large stars as supernovae), then the fluctuations in integrated brightness must correlate inversely with the absolute luminosity. This follows since the fluctuation in brightness must be proportional to the inverse square root of the total number of (similar) sources contributing to the brightness at any time.

Here again the results are suggestive (Figure 4). The amplitude-luminosity relation of the QSOs shows them separated into at least two and perhaps three discrete groups: the four violent variables, 3C 279, 3C 345, 3C 446 and 3C 454.3, are in a class by themselves at the top. The three objects (3C 120, Ton 730 and Ton 1542) indicated in Figure 4 by open circles, most resemble Seyfert galaxies. Their small redshifts imply luminosities four magnitudes fainter than the regular QSOs. These last, indicated by filled circles, form a group which, albeit with much scatter, does suggest the slope predicted by Wheeler's argument for multiple-source models, although the fit would be improved on the assumption that the more luminous QSOs also tend to be composed of brighter individual events.

\section{Reference}

Angione, R. J.: 1970, Dissertation, Astronomy Department, University of Texas at Austin (June, 1970); in preparation for publication. 